Merck, Roche, Pfizer, and UCB Pharma, Frank Buttgereit Grant/research support from: Amgen, BMS, Celgene, Generic Assays, GSK, Hexal, Horizon, Lilly, medac, Mundipharma, Novartis, Pfizer, Roche, and Sanofi.

DOI: 10.1136/annrheumdis-2020-eular.4391

\section{OP0301 PREDICTION OF LOW BONE MINERAL DENSITY AND FRAX SCORE BY ASSESSING HIP BONE TEXTURE WITH DEEP LEARNING}

C. F. Kuo ${ }^{1,2}$, S. $\mathrm{Miao}^{3}$, K. Zheng ${ }^{3}$, L. Lu ${ }^{3}$, C. I. Hsieh ${ }^{1}$, C. Lin ${ }^{2}$, T. Y. Fan ${ }^{2} .{ }^{1}$ Chang Gung Memorial Hospital, Division of Rheumatology, Allergy and Immunology, Taoyuan, Taiwan, Republic of China; ${ }^{2}$ Chang Gung Memorial Hospital, Center for Artificial Intelligence in Medicine, Taoyuan, Taiwan, Republic of China; ${ }^{3}$ PAII Labs, Bethesda, United States of America

Background: Osteoporosis is a widespread health concern associated with an increased risk of fractures in individuals with low bone mineral density (BMD). Dual-energy $x$-ray absorptiometry (DXA) is the gold standard to measure BMD, but methods based on the assessment of plain films, such as the digital radiogrammetry, ${ }^{1}$ are also available. We describe a novel approach based on the assessment of hip texture with deep learning to estimate BMD.

Objectives: To compare the BMD estimated by assessing hip texture using a deep learning model and that measured by DXA.

Methods: In this study, we identified 1,203 patients who underwent DXA of left hip and hip plain film within six months. The dataset was split into a training set with 1,024 patients and a testing set with 179 patients. Hip images were obtained and regions of interest (ROI) around left hips were segmented using a tool based on the curve Graph Convolutional Network. The ROls are processed using a Deep Texture Encoding Network (Deep-TEN) model, ${ }^{2}$ which comprises the first 3 blocks of Residual Network with 18 layers (ResNet-18) model followed by a dictionary encoding operator (Figure 1). The encoded features are processed using a fully connected layer to estimate BMD. Five-fold cross-validation was conducted. Pearson's correlation coefficient was used to assess the correlation between predicted and reference BMD. We also test the performance of the model to identify osteoporosis (T-score $\leq-2.5$ )

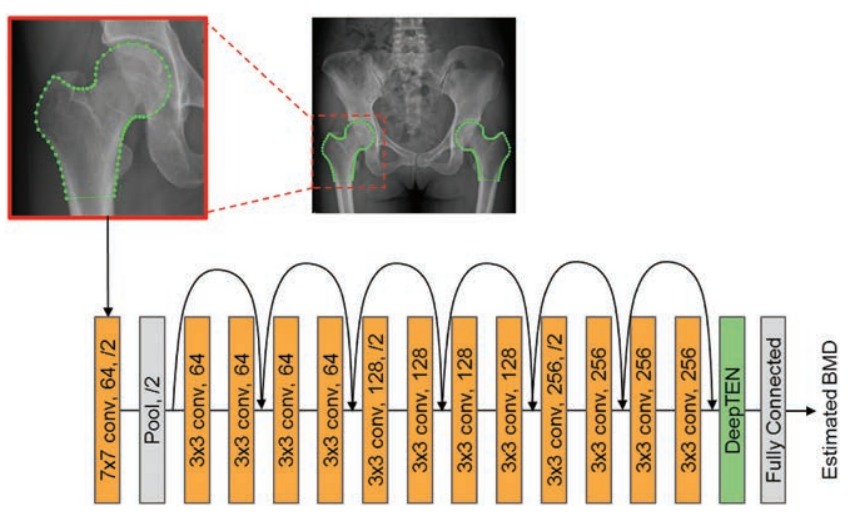

Figure 1. Schematic representation of deep learning models to extract and encode texture features for estimation of hip bone density.

Results: We included 151 women and 18 men in the testing dataset (mean age, $66.1 \pm 1.7$ years). The mean predicted BMD was $0.724 \mathrm{~g} / \mathrm{cm}^{2}$ compared with the mean BMD measured by DXA of $0.725 \mathrm{~g} / \mathrm{cm}^{2}(p=0.51)$. Pearson's correlation coefficient between predicted and true BMD was 0.88 . The performance of the model to detect osteoporosis/osteopenia was shown in Table 1. The positive predictive value was $87.46 \%$ for a T-score $\leq-1$ and $83.3 \%$ for a T-score $\leq-2.5$. Furthermore, the mean FRAX® 10-year major fracture risk did not differ significantly between scores based on predicted (6.86\%) and measured BMD $(7.67 \%$, $\mathrm{p}=0.52)$. The 10 -year probability of hip fracture was lower in the predicted score $(1.79 \%)$ than the measured score $(2.43 \%, p=0.01)$.

Table 1. Performance matrices of the deep texture model to detect osteoporosis/osteopenia

\begin{tabular}{lcc}
\hline & T-score $\leq-1$ & T-score $\leq-2.5$ \\
\hline Sensitivity & $91.11 \%$ & $33.33 \%$ \\
Specificity & $(95 \% \mathrm{Cl}, 83.23 \%$ to $96.08 \%)$ & $(95 \% \mathrm{Cl}, 17.29 \%$ to $52.81 \%)$ \\
& $86.08 \%$ & $98.56 \%$ \\
Positive predictive value & $(95 \% \mathrm{Cl}, 76.45 \%$ to $92.84 \%)$ & $(95 \% \mathrm{Cl}, 94.90 \%$ to $99.83 \%)$ \\
& $88.17 \%$ & $83.33 \%$ \\
Negative predictive value & $(95 \% \mathrm{Cl}, 81.10 \%$ to $92.83 \%)$ & $(95 \% \mathrm{Cl}, 53.58 \%$ to $95.59 \%)$ \\
& $89.47 \%$ & $87.26 \%$ \\
& $(95 \% \mathrm{Cl}, 81.35 \%$ to $94.31 \%)$ & $(95 \% \mathrm{Cl}, 84.16 \%$ to $89.83 \%)$ \\
\hline
\end{tabular}

Conclusion: This study demonstrates the potential of the bone texture model to detect osteoporosis and to predict the FRAX score using plain hip radiographs. References:

[1] Zandieh S, Haller J, Bernt R, et al. Fractal analysis of subchondral bone changes of the hand in rheumatoid arthritis. Medicine (Baltimore) 2017;96(11):e6344.

[2] Zhang H, Xue J, Dana K. Deep TEN: Texture Encoding Network. The IEEE Conference on Computer Vision and Pattern Recognition (CVPR) 2017:708-17.

Disclosure of Interests: None declared

DOI: 10.1136/annrheumdis-2020-eular.5916

\section{The new art of phenotyping and treating Sjögren's syndrome.}

\begin{tabular}{l|l}
\hline OP0302 & IANALUMAB (VAY736), A DUAL MODE OF ACTION \\
BIOLOGIC COMBINING BAFF RECEPTOR INHIBITION \\
WITH B CELL DEPLETION, REACHES PRIMARY \\
ENDPOINT FOR TREATMENT OF PRIMARY \\
SJOGREN'S SYNDROME
\end{tabular}

T. Dörner ${ }^{1}$, S. J. Bowman ${ }^{2}$, R. Fox ${ }^{3}$, X. Mariette ${ }^{4}$, A. Papas ${ }^{5}$, T. Grader-Beck ${ }^{6}$, B. A. Fisher ${ }^{2,7,8}$, F. Barcelos ${ }^{9}$, S. De Vita ${ }^{10}$, H. Schulze-Koops ${ }^{11}$, R. J. Moots ${ }^{12}$, G. Junge ${ }^{13}$, J. Woznicki ${ }^{14}$, M. Sopala ${ }^{13}$, W. L. Luo ${ }^{14}$, W. Hueber ${ }^{13} .{ }^{1}$ Department Medicine/Rheumatology and Clinical Immunology Charite Universitätsmedizin, Berlin, Germany; ${ }^{2}$ Department of Rheumatology, University Hospitals Birmingham NHS Foundation Trust, Birmingham, United Kingdom; ${ }^{3}$ Scripps Memorial Hospital and Research Institute, CA 92121, United States of America; ${ }^{4}$ Université Paris-Sud, Orsay, France; ${ }^{5}$ Division of Oral Medicine, Tufts School of Dental Medicine, Massachusetts, United States of America; ${ }^{6}$ Division of Rheumatology, Johns Hopkins School of Medicine, Baltimore, United States of America; ${ }^{7}$ University of Birmingham, Birmingham, United Kingdom; ${ }^{8} \mathrm{NIHR}$ Birmingham Biomedical Research Centre, Birmingham, United Kingdom; ${ }^{9}$ Instituto Português de Reumatologia, Lisbon, Portugal; ${ }^{10}$ Department of Medical Area, University of Udine, Udine, Italy; ${ }^{11}$ Division of Rheumatology and Clinical Immunology, Ludwig-Maximilians-University of Munich, Munich, Germany; ${ }^{12}$ Department of Musculoskeletal Biology, Aintree University Hospital, Liverpool, United Kingdom; ${ }^{13}$ Novartis Pharma AG, Basel, Switzerland; ${ }^{14}$ Novartis Pharmaceuticals Corporation, East Hanover, United States of America

Background: Primary Sjogren's syndrome (pSS) is a multi-organ autoimmune disease mainly affecting excretory glands and characterised by B-cell hyperactivity. No approved systemic treatment is available. lanalumab (VAY736) is an anti-B-cell activating factor (BAFF) receptor fully human monoclonal antibody, engineered for direct ADCC-mediated B-cell depletion.

Objectives: This phase $2 \mathrm{~b}$ study aimed at establishing a dose-response relationship over a range of VAY736 doses, using change from baseline (BL) in EULAR Sjogren's Syndrome Disease Activity index (ESSDAI) over 24 Weeks (Wks) as primary endpoint. The study is ongoing with a second blinded treatment period up to Wk52. Here we report efficacy and safety Wk24.

Methods: 190 patients (pts) were randomised 1:1:1:1 to receive monthly s.c. doses of VAY736 $(5,50,300 \mathrm{mg})$ or placebo (PBO). Prior to 1st-dose of study treatment, pts received methylprednisolone i.v. 250mg. Eligible pts fulfilled American European Consensus Group (AECG) criteria, were anti-Ro/SSA+, had ESSDAI $\geq 6$ and EULAR Sjogren's Syndrome Patient Reported Index (ESSPRI) $\geq 5$. Statistical methods included MCP-Mod to assess dose-response on change of ESSDAI from BL and responder rate analysis to calculate the proportion of pts with $\geq 3$ points improvement on ESSDAI. Secondary endpoints included ESSPRI, Functional Assessment of Chronic Illness Therapy Fatigue (FACIT-F), Physician's (PhGA) and Patient's Global Assessments (PaGA), SF-36, stimulated salivary flow (SSF), Schirmer's test.

Results: Primary endpoint was met with statistically significant dose-response for ESSDAI (Figure). The largest ESSDAI reduction was 1.92 points over PBO for VAY736 300mg at Wk24. Responder rate analysis on ESSDAI revealed for $300 \mathrm{mg}$ vs PBO responder rates of $42 / 47(89.4 \%)$ vs $30 / 49(61.2 \%)$, a difference of $28.1 \%(p=0.0019)$. No differences were seen for $5 \mathrm{mg}$ and $50 \mathrm{mg}$ vs PBO. PhGA change from BL was significantly different between $300 \mathrm{mg}$ and $\mathrm{PBO}$ $(p=0.022)$. A numerical trend for sSF improvement for VAY736 300mg compared to PBO was notable at Wk24 $(p=0.092)$. For secondary endpoints ESSPRI and FACIT-F, VAY736 treatment showed no benefits over PBO. PBO responses were generally high. Incidence of treatment emergent AEs was comparable across all studied groups, whereby site injection reactions were most frequent, mostly mild and showed a dose-response.

Conclusion: Primary endpoint assessing ESSDAI was met, showing statistically significant dose-response for ianalumab with clinically important improvement for $300 \mathrm{mg}$ vs PBO. Preliminary safety profile of ianalumab was good. 


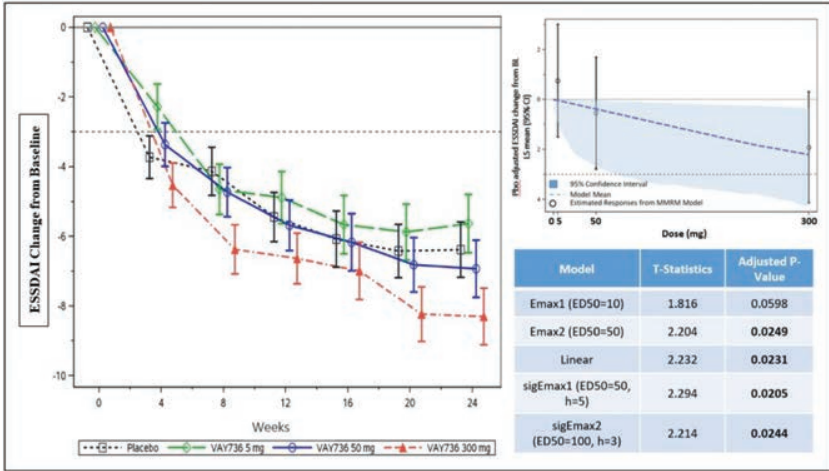

Figure. ESSDAI Change from Baseline over Time up to Week 24 Reveals a Statistically Significant Dose Response Relationship

Disclosure of Interests: Thomas Dörner Grant/research support from: Janssen, Novartis, Roche, UCB, Consultant of: Abbvie, Celgene, Eli Lilly, Roche, Janssen, EMD, Speakers bureau: Eli Lilly, Roche, Samsung, Janssen, Simon J. Bowman Consultant of: Astrazeneca, Biogen, BMS, Celgene, Medimmune, MTPharma, Novartis, Ono, UCB, xtlbio, Glapagos, Speakers bureau: Novartis, Robert Fox Consultant of: Novartis, Pfizer and Lilly, Xavier Mariette Consultant of: BMS, Gilead, Medimmune, Novartis, Pfizer, Servier, UCB, Athena Papas Grant/ research support from: Novartis, Consultant of: Novartis, Thomas Grader-Beck Grant/research support from: Abbvie, Celgene, Consultant of: Novartis, Lilly, Ben A Fisher Consultant of: Novartis, Roche, BMS and Servier, Filipe Barcelos Consultant of: Pfizer and Lilly, Salvatore De Vita Consultant of: Roche, Human Genome Science, Glaxo Smith Kline and Novartis, Hendrik Schulze-Koops Grant/research support from: Pfizer Inc, Robert J Moots: None declared, Guido Junge Shareholder of: Novartis, Employee of: Novartis, Janice Woznicki Shareholder of: Novartis, Employee of: Novartis, Monika Sopala Shareholder of: Novartis, Employee of: Novartis, Wen-Lin Luo Shareholder of: Novartis, Employee of: Novartis, Wolfgang Hueber Shareholder of: Novartis, Employee of: Novartis DOI: 10.1136/annrheumdis-2020-eular.364

\section{Meniscus : an innocent bystander in osteoarthritis?.}

\begin{tabular}{|l|l}
\hline OP0303 & CORTICOSTEROID INJECTION IN KNEE \\
OSTEOARTHRITIS IS ASSOCIATED WITH REDUCTION \\
IN MENISCAL THICKNESS AND JOINT SPACE WIDTH \\
WITH NO EFFECT ON CARTILAGE THICKNESS: A \\
CASE CONTROL STUDY
\end{tabular}

J. P. Pelletier ${ }^{1}$, J. P. Raynauld ${ }^{1}$, F. Abram ${ }^{2}$, M. Dorais ${ }^{3}$, P. Paiement ${ }^{4}$, J. MartelPelletier ${ }^{1}{ }^{1}$ University of Montreal Hospital Research Centre (CRCHUM), Osteoarthritis Research Unit, Montreal, Canada; ${ }^{2}$ ArthroLab Inc., Medical Imaging Research \& Development, Montreal, Canada; ${ }^{3}$ StatSciences Inc., Notre-Dame-de-l'île-Perrot, Canada; ${ }^{4}$ ArthroLab Inc., Montreal, Canada

Background: Intra-articular corticosteroid injections (IACI) are commonly used for the treatment of symptomatic knee osteoarthritis (OA) and therapeutic guidelines have recommended their use. However, their safety regarding the evolution of structural changes remains unknown.

Objectives: This study explored the effects of $I A C I$ on the evolution of knee OA structural changes assessed by magnetic resonance imaging (MRI).

Methods: Participants were selected from the Osteoarthritis Initiative database. In this nested case-control design study, participants who received one treatment with $\mathrm{IACl}$ and had MRI exams available at the yearly follow-up visits before (pre-treatment), during (treatment), and after (post-treatment) were defined as "cases". Each case was matched with one control for age, gender, body mass index (BMI), height, joint space width (JSW), cartilage volume, bone marrow lesion (BML), meniscal extrusion, and Western Ontario and McMaster Universities Osteoarthritis Index (WOMAC) pain at baseline. Ninety-three (93) participants fulfilling the inclusion criteria were selected and matched to controls $(n=93)$. The study structural variables were MRI (cartilage volume, meniscal thickness, bone marrow lesion (BML), bone curvature), X-rays (JSW), and symptoms (WOMAC pain), assessed at the yearly consecutive visits and changes measured within the follow-up periods.

Results: At baseline, the control and treatment groups were balanced. In the pre-treatment period, the cartilage volume loss in the medial compartment was significantly greater in the $I A C I(p=0.006)$ compared to the control group, with a numerical trend $(p=0.071)$ in the lateral compartment. In the treatment period, the cartilage loss was not different between groups, with the exception of a significantly greater loss in the lateral compartment in the IACI group $(p=0.041)$. In the post-treatment period there was no difference in the cartilage loss between the groups in both compartments. For the meniscal thickness loss in the pre-treatment period, no difference was found between groups; however there was a significantly greater loss $(p=0.007)$ during the treatment period in the $\mathrm{IACl}$. In the post-treatment period, the loss of the medial meniscus was similar in both groups. For the lateral meniscus, there was no significant difference at any time between the two groups. The loss in JSW in the pre- and post-treatment periods was not different between groups, but was significantly greater $(p=0.011)$ in the $\mathrm{IACl}$ group in the treatment period. The changes in the BML sizes over time were small and similar between groups. For the bone curvature, IACI group showed a smaller change compared to the control $(p=0.037)$ at the treatment period. The WOMAC pain changes in both groups were small and unlikely to be clinically relevant.

Conclusion: This study provides evidence that in knee $\mathrm{OA}, \mathrm{IACl}$ were not associated with the occurrence of any deleterious effect on knee structures post-treatment, including cartilage volume and loss. The increase in the rate loss of medial meniscal thickness, which was associated with a loss of JSW, was a transient phenomenon and its clinical relevance unknown at that time.

Acknowledgments: This initiative was funded by a grant from La Chaire en arthrose de l'Université de Montréal, and by ArthroLab Inc. (both in Montreal, Quebec, Canada).

Disclosure of Interests: Jean-Pierre Pelletier Shareholder of: ArthroLab Inc. Grant/research support from: TRB Chemedica, Speakers bureau: TRB Chemedica and Mylan, Jean-Pierre Raynauld Consultant of: ArthroLab Inc., François Abram Employee of: ArthroLab Inc., Marc Dorais Consultant of: ArthroLab Inc. Patrice Paiement Employee of: ArthroLab Inc., Johanne Martel-Pelletier Shareholder of: ArthroLab Inc., Grant/research support from: TRB Chemedica DOI: 10.1136/annrheumdis-2020-eular.1755

\section{SpA: Innovative techniques in spondyloarthritis research - Is there light at the end of the tunnel for our patients?}

\begin{tabular}{|l} 
OP0304 \\
METABOLOMICS PROFILING OF HUMAN SERUM \\
FOR DISCOVERING BIOMARKERS TO DIAGNOSE \\
PSORIATIC ARTHRITIS AND ANKYLOSING \\
SPONDYLITIS WITH HIGH SPECIFICITY
\end{tabular}

W. Han ${ }^{1}$, X. Wang ${ }^{1}$, L. LI ${ }^{1}$, S. Wichuk ${ }^{1}$, E. Hutchings ${ }^{2}$, R. Dadashova ${ }^{2}$,

J. Paschke ${ }^{2}$, W. P. Maksymowych ${ }^{2} .{ }^{1}$ University of Alberta, Edmonton, Canada; ${ }^{2}$ CARE Arthritis, Edmonton, Canada

Background: High-specificity biomarkers that can differentiate PsA and AS patients from healthy people, as well as RA patients, would be extremely helpful for early diagnosis and treatment of these two diseases. Based on the hypothesis that each disease state may cause specific changes to the metabolome metabolomics is becoming a powerful tool for biomarker discovery. In this work we applied a high-performance chemical isotope labeling (CIL) LC-MS platform to search for biomarker candidates of PsA and AS in human serum samples. Objectives: We aimed to identify metabolite biomarkers with high specificity for PsA and AS.

Methods: Serum samples were collected from 331 subjects, including 100 healthy controls, 48 PsA patients, 52 AS patients and 131 RA patients. The average age of each group was: 52.6 (control), 50.7 (PsA), 51.8 (AS) and 53.1 (RA) years. After pre-treatment, each sample was incubated with ${ }^{12} \mathrm{C}$-dansyl chloride, which can label the amine/phenol-containing metabolites. The reference sample for relative quantification was prepared by mixing all individual samples and then labeled by ${ }^{13} \mathrm{C}$-dansyl chloride. With this normalization, the individual samples and the reference sample were mixed at an equal amount. Finally, we used an LC-QTOF-MS platform to analyze the mixtures and measure the ${ }^{12} \mathrm{C} /{ }^{13} \mathrm{C}$ peak pairs.

Results: We detected 1,149 peak pairs commonly existing in the serum samples. Using our dansyl-library of 700 dansyl-labeled standards and a prediction library, which contains the predicted retention times and mass values of 3,431 dansylated human metabolites, we identified 134 and 141 peak pairs, respectively. The relative concentrations are calculated from the intensity ratios of ${ }^{12} \mathrm{C} /{ }^{13} \mathrm{C}$ peak pairs. We first visualized the entire amine/phenol-submetabolome for all phenotypes using the partial least squares discriminant analysis (PLS-DA). We found that the most significant between-group separation was between healthy controls and all the patients. No significant sex or age effect was observed. Furthermore, among the three diseases, PsA and AS samples were closely clustering, while the RA group was well separated from them. Therefore, we chose a two-step diagnosis approach that first differentiates PsA patients from controls/RA patients and then filters out the AS patients wrongly classified as PsA in the first step. The same strategy was conducted for AS. Stipulating a fold change larger than 1.5 with the false discovery rate lower than $5 \%$, we found 74 metabolites having significantly 